University of Nebraska - Lincoln

DigitalCommons@University of Nebraska - Lincoln

USDA National Wildlife Research Center - Staff Publications
U.S. Department of Agriculture: Animal and Plant Health Inspection Service

2021

\title{
Variation in Angiostrongylus cantonensis infection in definitive and intermediate hosts in Hawaii, a global hotspot of rat lungworm disease
}

\author{
Chris Niebuhr \\ USDA, APHIS, Wildlife Services, National Wildlife Research Center, niebuhrc@landcareresearch.co.nz \\ Shane R. Siers \\ USDA APHIS Wildlife Services, shane.r.siers@aphis.usda.gov \\ Israel Leinbach \\ USDA APHIS Wildlife Services NWRC, israel.I.leinbach@usda.gov \\ Lisa M. Kaluna \\ University of Hawaii at Hilo \\ Susan I. Jarvi

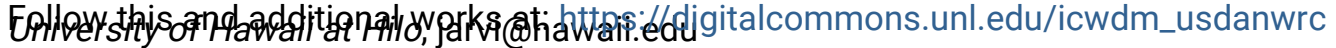 \\ Part of the Natural Resources and Conservation Commons, Natural Resources Management and \\ Policy Commons, Other Environmental Sciences Commons, Other Veterinary Medicine Commons, \\ Population Biology Commons, Terrestrial and Aquatic Ecology Commons, Veterinary Infectious Diseases \\ Commons, Veterinary Microbiology and Immunobiology Commons, Veterinary Preventive Medicine, \\ Epidemiology, and Public Health Commons, and the Zoology Commons
}

Niebuhr, Chris; Siers, Shane R.; Leinbach, Israel; Kaluna, Lisa M.; and Jarvi, Susan I., "Variation in Angiostrongylus cantonensis infection in definitive and intermediate hosts in Hawaii, a global hotspot of rat lungworm disease" (2021). USDA National Wildlife Research Center - Staff Publications. 2414.

https://digitalcommons.unl.edu/icwdm_usdanwrc/2414

This Article is brought to you for free and open access by the U.S. Department of Agriculture: Animal and Plant Health Inspection Service at DigitalCommons@University of Nebraska - Lincoln. It has been accepted for inclusion in USDA National Wildlife Research Center - Staff Publications by an authorized administrator of DigitalCommons@University of Nebraska - Lincoln. 
Parasitology

cambridge.org/par

\section{Research Article}

Cite this article: Niebuhr CN, Siers SR Leinbach IL, Kaluna LM, Jarvi SI (2021). Variation in Angiostrongylus cantonensis infection in definitive and intermediate hosts in Hawaii, a global hotspot of rat lungworm disease. Parasitology 148, 133-142. https:// doi.org/10.1017/S003118202000164X

Received: 1 June 2020

Revised: 21 August 2020

Accepted: 22 August 2020

First published online: 10 September 2020

\section{Key words:}

Angiostrongyliasis; drivers; eosinophilic meningitis; nematode; parasite; Parmarion; Rattus; slug; snail; transmission

Author for correspondence:

Chris N. Niebuhr,

E-mail: niebuhrc@landcareresearch.co.nz

\section{Variation in Angiostrongylus cantonensis infection in definitive and intermediate hosts in Hawaii, a global hotspot of rat lungworm disease}

\author{
Chris N. Niebuhr ${ }^{1,2}$ (D), Shane R. Siers ${ }^{1}$, Israel L. Leinbach ${ }^{1}$, Lisa M. Kaluna ${ }^{3}$ \\ and Susan I. Jarvi ${ }^{3}$ (iD
}

${ }^{1}$ USDA APHIS Wildlife Services, National Wildlife Research Center, Hawaii Field Station, Hilo, HI, USA; ${ }^{2}$ Manaaki Whenua-Landcare Research, PO Box 69040, Lincoln 7640, New Zealand and ${ }^{3}$ Department of Pharmaceutical Sciences, Daniel K. Inouye College of Pharmacy, University of Hawaii at Hilo, Hilo, HI, USA

\begin{abstract}
Angiostrongylus cantonensis (rat lungworm) is a tropical and subtropical parasitic nematode, with infections in humans causing angiostrongyliasis (rat lungworm disease), characterized by eosinophilic meningitis. Hawaii has been identified as a global hotspot of infection, with recent reports of high infection rates in humans, as well as rat definitive and snail intermediate hosts. This study investigated variation in A. cantonensis infection, both prevalence and intensity, in wild populations of two species of rats (Rattus exulans and R. rattus) and one species of snail (Parmarion martensi). An overall infection prevalence of $86.2 \%$ was observed in $P$. martensi and $63.8 \%$ in rats, with $R$. exulans (77.4\%) greater than $R$. rattus (47.6\%). We found infections to vary with environmental and host-related factors. Body mass was a strong predictor of infection in all three species, with different patterns seen between sexes and species of rats. Infection prevalence and intensity for $R$. exulans were high in May 2018 and again in February 2019, but generally lower and more variable during the intervening months. Information on sources of variability of infection in wild host populations will be a crucial component in predicting the effectiveness of future disease surveillance or targeted management strategies.
\end{abstract}

\section{Introduction}

Angiostrongylus cantonensis (Nematoda: Metastrongylidae), or the rat lungworm, is the primary aetiologic agent of eosinophilic meningitis in humans worldwide, with recent reports of increased human infection, geographic spread and host expansion (Wang et al., 2008; Graeff-Teixeira et al., 2009; York et al., 2015; Niebuhr et al., 2020). These recent increases in human cases, as well as high A. cantonensis infection rates observed in wildlife, have identified Hawaii as a global hotspot of infection, while simultaneously highlighting gaps in knowledge surrounding local pathways for transmission (Cowie, 2013; Jarvi et al., 2018; Johnston et al., 2019; Niebuhr et al., 2019). Perhaps surprisingly, as it was early research conducted in the 1960s in Hawaii that led to the first link between A. cantonensis and human disease (angiostrongyliasis, or rat lungworm disease) (Alicata, 1991), our current understanding of host-parasite transmission dynamics remains limited in Hawaii and elsewhere (Prociv et al., 2000; Qvarnstrom et al., 2013; Jarvi et al., 2017; Kim et al., 2019).

The life cycle of the parasite is complex, requiring both rats definitive and gastropod intermediate hosts to reproduce. Infected gastropods, a common source of human and other animal infection, allow for early larval development, while the rat host is required for the sexual reproduction of the parasite (Spratt, 2015; Barratt et al., 2016). Infections of A. cantonensis in wild host populations are highly variable (Wang et al., 2008; Barratt et al., 2016) and are influenced by a variety of factors, both intrinsic (e.g. host species, size, diet) and extrinsic (e.g. location, climate, seasonality) (Ibrahim, 2007; Kim et al., 2014, 2019; Simões et al., 2014; Jarvi et al., 2017).

In Hawaii, all three species of introduced rats (Rattus exulans, R. rattus and R. norvegicus) and a variety of native and non-native snails are known to harbour A. cantonensis infections (Kim et al., 2014; Niebuhr et al., 2019). One gastropod species, in particular, Parmarion martensi, often carries higher $A$. cantonensis infection levels (prevalence and intensity) than other gastropods (Hollingsworth et al., 2007; Qvarnstrom et al., 2013; Kim et al., 2014) and may be more easily infected (Asato et al., 2004). The recent spread of this introduced terrestrial snail sometimes referred to as a semi-slug, may help explain the increased incidence of human cases seen in both Hawaii and Japan (Asato et al., 2004; Howe and Jarvi, 2017). While infection levels of $A$. cantonensis in wild host populations have been previously investigated in Hawaii, often interpretations or comparisons of findings are based on limited representation in terms of locality, seasonality, or various host characteristics. The aim of this study was to investigate intrinsic and extrinsic factors associated with variation in A. cantonensis infections
(C) The Author(s), 2020. This is a work of the U.S. Government and is not subject to copyright protection in the United States. Published by Cambridge University Press. 
in two definitive and one intermediate host species from shared habitats in eastern Hawaii Island.

\section{Materials and methods}

\section{Study area and host collection}

In 2018-2019, wild rats ( $R$. exulans and $R$. rattus) and snails $(P$. martensi) were sampled for $A$. cantonensis infection. Specimens were collected from two sites in Hilo, on the eastern side of Hawaii Island, Hawaii, USA, an area previously reported to have high infection levels in all three species studied here (Qvarnstrom et al., 2013; Jarvi et al., 2017). The two sites, Site $1\left(19^{\circ} 42^{\prime} 47^{\prime \prime} \mathrm{N} 155^{\circ} 03^{\prime} 11^{\prime \prime} \mathrm{W}\right)$ and Site $2\left(19^{\circ} 39^{\prime} 12^{\prime \prime} \mathrm{N}\right.$ $155^{\circ} 04^{\prime} 25^{\prime \prime} \mathrm{W}$ ) are less than $500 \mathrm{~m}$ from the airport and the local zoo, respectively. The two sites are approximately $7 \mathrm{~km}$ apart and situated at 25 and $120 \mathrm{~m}$ elevation a.s.l. Site 1 is less than $500 \mathrm{~m}$ from the airport and Site 2 is less than $300 \mathrm{~m}$ from the local zoo. This overall region has a tropical rainforest climate with considerable rainfall occurring throughout the year (annual mean of 3219 and $3982 \mathrm{~mm}$ for each site) and a mean annual temperature at each site of 23.3 and $21.4^{\circ} \mathrm{C}$, respectively (NNDC CDO, 2020). Host collection took place over 12 months, which we divided into four sampling periods: Period A (early May for Site 1 and mid-May for Site 2, 2018), Period B (mid-August for Site 1 and early September for Site 2, 2018), Period C (late November for Site 1 and early December for Site 2, 2018), and Period D (mid-February for Site 1 and late February for Site 2, 2019); exact dates are included in Supplementary Table S1. Each collection event occurred over a period of 2-10 days.

Rats were collected using wire cage live traps (Haguruma, Osaka, Japan) baited with coconut and placed along transects at $10 \mathrm{~m}$ intervals. Each trapping site consisted of two transects, approximately $500 \mathrm{~m}$ apart. Transects were typically along forest or other vegetation edges, with traps, placed a few metres into the vegetation. Following capture, rats were euthanased via $\mathrm{CO}_{2}$ immersion and either immediately sampled for A. cantonensis or frozen $\left(-20^{\circ} \mathrm{C}\right)$ and sampled at a later date. Snails were collected opportunistically within vegetation, or from underneath plastic tarps previously placed on the ground, and subsequently frozen $\left(-80^{\circ} \mathrm{C}\right)$ for later analysis.

\section{Angiostrongylus in wild rats}

Sex and body mass of each rat was recorded, along with species, which is easily distinguishable using morphological characteristics (Innes, 1990). The heart and lungs of each rat were then removed and inspected using fine-tipped forceps under a stereomicroscope (Leica or Olympus 10-40X). The lung texture of each rat was inspected for a granulated appearance, as discussed by Jarvi et al. (2017). Adult stage worms were collected and counted from the right ventricle, pulmonary artery and lungs of each rat. Lungworm species identification was confirmed by Červená et al. (2019) and voucher specimens were deposited in the Australian National Wildlife Collection (CSIRO, Canberra, Australian Capital Territory, Australia) under accession number W/L HC\# N5703-N5711. The complete mtDNA genome sequence of a voucher specimen was submitted to GenBank under accession number MK570630.

\section{Angiostrongylus in P. martensi}

Prior to molecular analysis, each snail was thawed, weighed and measured for length. The length was measured by positioning each snail on their ventral side as straight as possible, without forcibly extending the body outwards; due to this species having an underdeveloped shell, the entire body was accessible. Tissue samples (ranging from 10 to $307 \mathrm{mg}$; mean of $110.3 \pm 3.8$ S.E.), were dissected from the tail region, as this region is known to harbour consistently high larval burdens of $A$. cantonensis in $P$. martensi (Jarvi et al., 2012). Tissue samples were stored in $500 \mu \mathrm{L}$ of DNA lysis buffer and homogenised using a Bead Ruptor Elite (Omni International, Kennesaw, Georgia, USA) as described in Niebuhr et al. (2020). DNA was extracted from $50 \mu \mathrm{L}$ of homogenised tissue using the DNeasy ${ }^{\circ}$ Blood \& Tissue Kit (QIAGEN, Valencia, California, USA) per the manufacturer's Animal Tissue protocol with a final elution volume of $400 \mu \mathrm{L}$.

Quantification of $A$. cantonensis (number of larvae per reaction tube) from tail tissues of snails was estimated by quantitative PCR (qPCR) using the assay described by Jarvi et al. (2012), the 50 -cycle cycling conditions outlined by Niebuhr et al. (2020), $9 \mu \mathrm{L}$ of extracted template and the plasmid standards developed by Howe et al. (2019). While only tail tissue was analysed here, to allow for more consistency when making comparisons, the quantification of parasites found should not necessarily be extrapolated to the rest of the body, as A. cantonensis may not be evenly distributed throughout the entire snail (Jarvi et al., 2012). Confidence in the quantification estimates of each qPCR run was established by high qPCR efficiencies (Bustin et al., 2009) and that the standard curves of reference samples encompassed the cycle threshold $(\mathrm{Ct})$ range observed in all snail samples. Confidence in the quantification estimates of individual snail samples was established by a low cycle threshold standard deviation (Ct s.D.) $(<0.5)$ among replicates within a qPCR run. Samples were classified as positive or negative using the criteria outlined in Niebuhr et al. (2020). For positive samples, the amount of tissue used in each DNA extraction (tissue amount assayed) and the intensity of infection (estimated quantity of larvae per mg of tissue) was determined by the two following equations:

Tissue amount assayed $(\mathrm{mg})=($ tissue amount dissected $(\mathrm{mg}) /$ $500 \mu \mathrm{L}$ DNA lysis buffer) $\times 50 \mu \mathrm{L}$ homogenised tissue used for DNA extraction

Estimated quantity of larvae per mg of tissue $=((\#$ of larvae per reaction tube $/ 9 \mu \mathrm{L}$ extracted template $) \times 400 \mu \mathrm{L}$ final elution $) /$ tissue amount assayed (mg)

\section{Data analysis}

We investigated the effects of intrinsic factors (rat body mass and sex; snail body length or mass) and extrinsic factors (site and sampling period) on A. cantonensis infection. For rats, infection prevalence (infection status: positive/negative) was assessed by multiple logistic regression, i.e. generalized linear models (GLMs) with a binomial distribution and logit link function. Infection intensity (total number of adult worms observed from each rat) was evaluated with GLMs with a negative binomial distribution. For snails, prevalence (infection status: positive/negative) was assessed with logistic regression. Infection intensity in snails was modelled as the estimated quantity of larvae per mg of tissue; because these values were not true counts, they were logtransformed (natural $\log$ ) to improve normality and intensity was evaluated by linear regression.

Potential intrinsic predictor terms included mass, sex and a sex $\times$ mass interaction for rats, and mass or length as alternative predictors for snails. Extrinsic predictors of site (1 and 2), sampling period $(\mathrm{A}-\mathrm{D})$ and a site $\times$ period interaction term were evaluated for both rats and snails. For mass and length terms, we also considered a quadratic term (mass ${ }^{2}$ or length ${ }^{2}$ ); quadratic regression allows for a better fit to data resulting from a non-linear response, for example, a curvilinear relationship between mass and prevalence or intensity (e.g. a 'humped' or 'U-shaped' 
response where prevalence or intensity is higher for mid-sized individuals than for smaller and larger ones). Models were constrained to always include the lower-order terms (main effects) when including interactions or quadratic terms, e.g. all models with a mass ${ }^{2}$ term were constrained to also include the mass term $\left(\right.$ mass + mass $^{2}$ ), and all models with interactions, e.g. sex $\times$ mass, were constrained to also include both of the main effects (sex + mass + sex $\times$ mass). For rats, exploratory modelling showed no meaningful sex $\times$ site or sex $\times$ period effects, so these interaction terms were not included in the model selection process. Because snail length and mass are highly autocorrelated (adjusted $R^{2}=0.449, P<<0.001$ ), model sets were constrained to include only length or mass, never both.

Combinations of potential predictor variables were evaluated for fit to the data using Akaike's Information Criteria corrected for small sample size $\left(\mathrm{AIC}_{C}\right)$, with weights assigned to all candidate models as per Burnham and Anderson (2002). The model selection included all combinations of potential predictors within the constraints described above. Models within $2 \mathrm{AIC}_{C}$ units of the top model were considered plausible alternative models. Our tables of model selection results include only: all models within $2 \mathrm{AIC}_{C}$ units of the top model (plausible top models, in bold); models including each of the main effects alone; and the intercept-only (null) model - for the sake of brevity, models containing other potential combinations of terms were considered but not listed. When the mass + mass $^{2}$ model outperformed the mass-only model, the superior model was reported because mass and mass ${ }^{2}$ are in fact a single effect. For each effect term, the relative variable importance (RVI; Anderson, 2008) or the summed weights of all models containing that term, was calculated; e.g. an RVI of 1.0 indicates that the respective term was included in models carrying $100 \%$ of model weights and lower values indicate relatively less combined weight in the model set (lower importance of the variable). All statistical analyses were run in $\mathrm{R}$ version 3.5.3 ( $\mathrm{R}$ Core Team, 2019) and multi-model inference was performed with functions in the 'MuMIn' package.

\section{Results}

\section{Infection in rats}

A total of 417 rats (226 R. exulans and 191 R. rattus) were caught from two sites in Hilo, Hawaii. No R. norvegicus were caught during this study. For each species, approximately $55 \%$ of rats caught were male. The mean body mass of $R$. exulans individuals caught was $48.8 \mathrm{~g}$ ( \pm 1.0 S.E.), with a minimum and maximum of 16.2 and $99.5 \mathrm{~g}$. For $R$. rattus, the mean body mass was $103.0 \mathrm{~g}$ ( \pm 2.7 s.e.), with a minimum and maximum of 21.7 and $186.0 \mathrm{~g}$. In total, 2,507 adult $A$. cantonensis were collected from 266 rats $(63.8 \%$ of 417 rats sampled). More R. exulans (77.4\% of 226 rats) were infected with adult $A$. cantonensis than $R$. rattus $(47.6 \%$ of 191 rats; $P<0.01$, Fisher's exact test) and those infected harboured more worms (mean of $11.1 \pm 0.7$ S.E.), than $R$. rattus (mean of $6.2 \pm 0.7$ S.E.; $P<0.0001$, unpaired, two-tailed $t$-test). Infection values by species, site and sampling period are included in Supplementary Tables S2-S3. The highest worm burden observed was 57 ( 32 males and 25 females) from a $63.6 \mathrm{~g}$ male $R$. exulans. The most worms observed in a $R$. rattus individual were 36 (13 males and 20 females) from an $80.0 \mathrm{~g}$ male rat. Additionally, $76.7 \%$ (204 of 266) of infected rats were observed to have granulation on at least one lung lobe (often the upper right).

\section{Infection prevalence in $\mathrm{R}$. rattus}

Mass and sex were the most important predictors of the presence of adult worms in $R$. rattus (RVI 0.99 and 0.93, respectively)
(Tables 1A and 2). The quadratic term for mass was also included in models carrying $71 \%$ of summed model weights. Sex $\times$ mass and sex $\times$ mass $^{2}$ interactions were only moderately important on their own (0.58 and 0.43) though at least one of these interactions was included in all 10 models within $3.359 \mathrm{AIC}_{C}$ units of the top model, comprising $78 \%$ of the summed model weights (includes models not shown in our reduced tables). There was little support for a strong influence of site, sampling period, or a site $\times$ period interaction $(0.44,0.20$ and 0.03$)$. In general, there was little ability to distinguish among models, with all model weights being relatively low. For heuristic value, we plot the second model to examine the influences of sex and mass (sex + mass + mass $^{2}+\operatorname{sex} x$ mass ${ }^{2}$; included in both top models) and site (the most important extrinsic predictor of prevalence) (Fig. 1A). This figure depicts a general trend of high prevalence in smaller males with a much lower prevalence in the largest males and a slightly increasing trend of infection with an increased mass in females. Smaller males were more likely to be infected than smaller females and larger females were more likely to be infected than larger males. Infection prevalence was only slightly higher at Site 2 .

\section{Infection prevalence in R. exulans}

Mass and mass ${ }^{2}$ were highly significant predictors of infection prevalence for R. exulans (RVI 1.0 for both), while sex was of relatively less importance (0.45) (Tables $1 \mathrm{~B}$ and 2). Interactions of sex with mass or mass ${ }^{2}$ were unimportant ( 0.13 for both), meaning the relationship between mass and prevalence was generally very similar for both sexes. In contrast to $R$. rattus, extrinsic factors (site and sampling period) were important predictors of prevalence in $R$. exulans ( 0.98 and 1.0 ), with a moderately important site by sampling period interaction (0.57). Compared to $R$. rattus, there was a greater ability to differentiate among models, with only two models within $2 \mathrm{AIC}_{C}$ units of the top model and the top three model weights summing to $66.5 \%$ of all model weights.

For comparison to $R$. rattus results, we also plotted prevalence predictions by sex, mass, mass ${ }^{2}$ and site (Fig. 1B). In this figure, we see the strong quadratic effects of mass + mass $^{2}$ on prevalence, with a significantly lower prevalence in very small and very large individuals, and very high prevalence for $R$. exulans from approximately 40 to $85 \mathrm{~g}$. Standard error intervals beyond $85 \mathrm{~g}$ are very broad due to the low number of rats $(n=3)$ in this size range. The effect of sex was relatively minor and we did not plot sex by mass or mass ${ }^{2}$ interaction because the first model containing such an interaction (not reported in our reduced model table) was $3.514 \mathrm{AIC}_{C}$ units greater than the top model. The site, sampling period and site $\times$ period carried RVI values of $0.98,1.0$ and 0.57; however, because of some categorical values equalling 1 (100\% prevalence), regression models could not estimate standard errors for sampling period effects. Rather, we plot the $95 \%$ binomial confidence intervals (exact method) for the prevalence of infection for all males and females (irrespective of mass) by site and sampling period (Fig. 2). This figure represents the proportion of $R$. exulans collected at the respective site and sampling period that were positive for rat lungworm infection; however, this site and sampling period plot does not control for body masses of the rats sampled. In other words, in regards to Fig. 2, we acknowledge that infection prevalence is also influenced by the size of the rat (as seen in Fig. 1B) and that the average size of rats captured at any time and place can vary.

\section{Infection intensity in $\mathrm{R}$. rattus}

Mass was the most important predictor of infection intensity in $R$. rattus (RVI 0.99), with mass ${ }^{2}$ having relatively little influence (0.37) (Tables 2 and $3 A$ ). Sex was relatively unimportant (0.33), with the sex-only model ranking more poorly than the 
Table 1. Model selection tables for the prevalence of Angiostrongylus cantonensis infection in rats (Rattus rattus and R. exulans) and snails (Parmarion martensi) from two sites over four sampling periods in Hilo, Hawaii.

\begin{tabular}{|c|c|c|c|c|c|}
\hline Model & $\mathrm{df}$ & logLik & $\mathrm{AIC}_{\mathrm{C}}$ & $\Delta \mathrm{AIC}_{C}$ & weight \\
\hline \multicolumn{6}{|l|}{ (A) Rattus rattus } \\
\hline sex + mass + mass $^{2}+$ sex $\times$ mass $^{2}$ & 5 & -123.60 & 257.52 & 0.00 & 0.148 \\
\hline sex + mass + mass $^{2}+$ sex $\times$ mass $^{2}+$ site & 6 & -122.81 & 258.07 & 0.55 & 0.113 \\
\hline sex + mass + mass $^{2}+\operatorname{sex} \times$ mass & 5 & -124.00 & 258.33 & 0.81 & 0.099 \\
\hline sex + mass + sex $\times$ mass + site & 5 & -124.05 & 258.42 & 0.90 & 0.095 \\
\hline sex + mass + sex $\times$ mass & 4 & -125.11 & 258.44 & 0.92 & 0.094 \\
\hline sex + mass + mass $^{2}+$ sex $\times$ mass + site & 6 & -123.32 & 259.10 & 1.58 & 0.067 \\
\hline sex + mass + mass $^{2}+$ sex $\times$ mass + sex $\times$ mass $^{2}$ & 6 & -123.47 & 259.40 & 1.88 & 0.058 \\
\hline mass + mass $^{2}$ & 3 & -127.56 & 261.25 & 3.72 & 0.023 \\
\hline period & 4 & -128.68 & 265.58 & 8.05 & 0.003 \\
\hline site & 2 & -131.00 & 266.05 & 8.53 & 0.002 \\
\hline (null) & 1 & -132.18 & 266.38 & 8.86 & 0.002 \\
\hline sex & 2 & -131.80 & 267.67 & 10.15 & 0.001 \\
\hline \multicolumn{6}{|l|}{ (B) Rattus exulans } \\
\hline mass + mass $^{2}+$ site + period + site $\times$ period & 10 & -48.63 & 118.28 & 0.00 & 0.316 \\
\hline mass + mass $^{2}+$ site + period & 7 & -52.25 & 119.00 & 0.73 & 0.219 \\
\hline sex + mass + mass $^{2}+$ site + period + site $\times$ period & 11 & -48.41 & 120.04 & 1.77 & 0.130 \\
\hline mass + mass $^{2}$ & 3 & -77.56 & 161.23 & 42.95 & 0.000 \\
\hline period & 4 & -79.51 & 167.20 & 48.92 & 0.000 \\
\hline (null) & 1 & -120.68 & 243.38 & 125.10 & 0.000 \\
\hline site & 2 & -119.84 & 243.72 & 125.45 & 0.000 \\
\hline Sex & 2 & -120.14 & 244.33 & 126.06 & 0.000 \\
\hline \multicolumn{6}{|l|}{ (C) Parmarion martensi } \\
\hline mass + site + period + site $\times$ period & 9 & -28.45 & 76.10 & 0.00 & 0.713 \\
\hline mass & 2 & -45.25 & 94.57 & 18.47 & 0.000 \\
\hline length & 2 & -47.30 & 98.67 & 22.57 & 0.000 \\
\hline period & 4 & -59.54 & 127.35 & 51.25 & 0.000 \\
\hline site & 2 & -62.07 & 128.22 & 52.12 & 0.000 \\
\hline (null) & 1 & -63.92 & 129.86 & 53.76 & 0.000 \\
\hline
\end{tabular}

The best models $\left(\Delta \mathrm{AIC} \mathrm{C}_{C} \leqslant 2\right)$ are highlighted in bold.

Tables include models that are within $2 \mathrm{AlC}_{\mathrm{c}}$ units of the top model (plausible alternative top models, in bold), models including each of the predictor terms alone, and the intercept-only (null) model with no predictor variables; additional combinations of terms were considered, but were unimportant and are not included here for the sake of brevity. Weight = the weight of the respective model in proportion to the sum of all model weights (1.0; Burnham and Anderson, 2002).

intercept-only (null model) and interactions of sex with mass or mass $^{2}$ were unimportant $(0.08$ and 0.03$)$. There was a moderate effect of the site (0.46), only when taking mass into account (i.e. the site-only model ranked more poorly than the null model). Neither sampling period nor site $\times$ period was influential $(0.17$ and $<0.01)$. The plausible top models (within $2 \mathrm{AIC}_{C}$ units of the top model) all contained mass in combination with mass ${ }^{2}$ and/or site. For comparison to infection prevalence plots, Fig. 1C shows the predicted worm intensity in $R$. rattus from the best-fit model that included sex, mass and site effects (sex + mass + mass $^{2}$ + site, not reported in our reduced table). From this figure we see that mean predicted infection intensity in $R$. rattus is primarily influenced by mass, with only a very slight curvature of the response curve due to the mass ${ }^{2}$ term and no difference by sex. Infection intensity at Site 2 was slightly lower (though with very broadly overlapping confidence intervals), in contrast to prevalence which was higher at Site 2.

\section{Infection intensity in R. exulans}

The most influential predictor of infection intensity in R. exulans was mass, with a moderately-important curvilinear relationship based on mass ${ }^{2}$ (RVI 1.0 and 0.73) (Tables 2 and 3B). Sex had a relatively low predictive influence (0.38) and only in combination with other terms, as the sex-only model ranked more poorly than the null model; no model including any form of the sex term was included in any of the plausible top models. Interactions of sex with mass and mass ${ }^{2}$ were unimportant $(0.11$ and 0.09$)$. As with prevalence in $R$. exulans, there was also a strong influence of sampling period on infection intensity $(0.88)$. The site was moderately influential (0.55), but only in models that also included an additive or interactive effect of the sampling period (the site-only model ranked more poorly than the null model) and the top model containing site without a sampling period effect (mass + mass $^{2}+$ site) performed more poorly than the top model by $4.64 \mathrm{AIC}_{C}$ units. There was only modest support for 
Table 2. Summary of relative variable importance (RVI, sum of model weights including the respective term; Anderson, 2008) for infection prevalence and intensity model sets.

\begin{tabular}{|c|c|c|c|c|c|c|c|c|c|}
\hline \multirow[b]{2}{*}{ Species } & \multirow[b]{2}{*}{ Infection level } & \multicolumn{8}{|c|}{ RVI of model term } \\
\hline & & sex & mass & mass $^{2}$ & sex $\times$ mass & sex $\times$ mass $^{2}$ & site & period & site $\times$ period \\
\hline \multirow[t]{2}{*}{ R. rattus } & Prevalence & 0.93 & 0.99 & 0.71 & 0.58 & 0.43 & 0.44 & 0.2 & 0.03 \\
\hline & Intensity & 0.33 & 0.99 & 0.37 & 0.08 & 0.03 & 0.46 & 0.17 & $<0.01$ \\
\hline \multirow[t]{3}{*}{ R. exulans } & Prevalence & 0.45 & 1 & 1 & 0.13 & 0.13 & 0.98 & 1 & 0.57 \\
\hline & Intensity & 0.38 & 1 & 0.73 & 0.11 & 0.09 & 0.55 & 0.88 & 0.38 \\
\hline & & mass & mass $^{2}$ & length & length $^{2}$ & site & period & site $\times$ period & \\
\hline \multirow[t]{2}{*}{ P. martensi } & Prevalence & 1 & 0.27 & $<0.01$ & $<0.01$ & 0.98 & 1 & 0.98 & \\
\hline & Intensity & 0.83 & 0.2 & 0.11 & 0.04 & 0.99 & 1 & 0.97 & \\
\hline
\end{tabular}
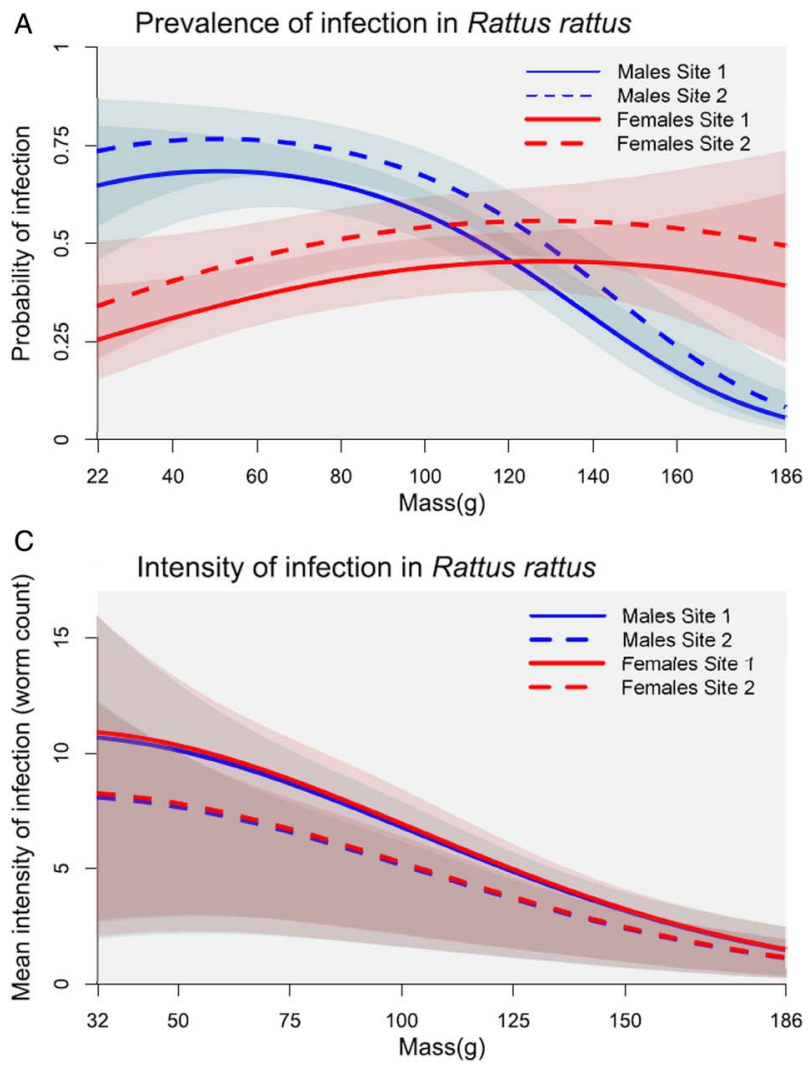

B Prevalence of infection in Rattus exulans
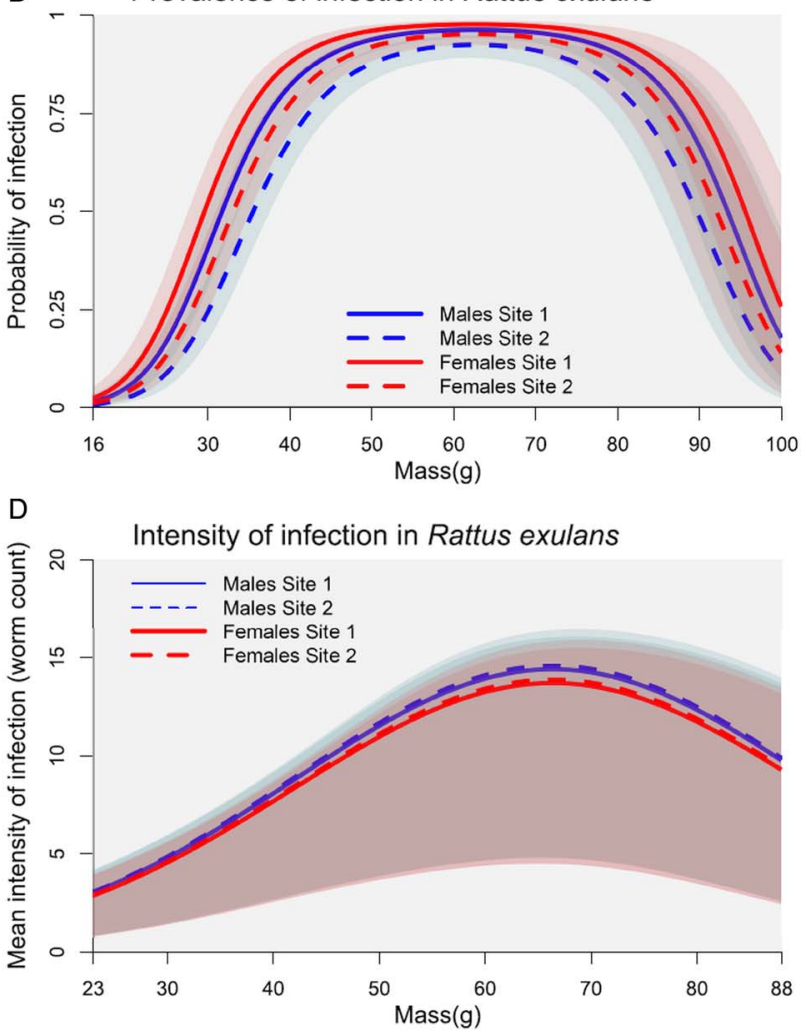

Fig. 1. Model predictions for relationships among sex, mass and site for prevalence and intensity of Angiostrongylus cantonensis infection in Rattus rattus (A \& C) and $R$. exulans (B \& D) sampled at two sites in Hilo, Hawaii. Shaded areas represent \pm 1 standard error for the estimate; where error intervals broadly overlap, there is little effect size.

an interaction between site and sampling period (0.38), though the interaction does appear in two of the top five plausible models.

Plotting effects of sex, mass and site, for comparison to previous plots (Fig. 1D), we see there is clearly very little influence of sex and almost no influence of site, on infection intensity in $R$. exulans. Here infection is clearly dominated by a mostly positive relationship with mass, though a mass ${ }^{2}$ influence results in a slight reduction in intensity for the largest rats (but with very broad confidence intervals). We also plot the sampling period effect from the top model, including mass and mass ${ }^{2}$ (Fig. 3). Controlling for mass, we see that infection intensity was highest in Periods A and D, with intensity lower in Periods $\mathrm{B}$ and $\mathrm{C}$.

\section{Infections in snails}

In total, 159 P. martensi snails were sampled, ranging in body mass from 0.05 to $2.36 \mathrm{~g}$ (mean of $1.08 \pm 0.04$ S.E.) and $1.20-$ $5.40 \mathrm{~cm}$ length (mean of $3.40 \pm 0.07$ S.E.). Overall, $86.2 \%$ (137 of $159)$ of snails were positive for A. cantonensis infection. The estimated quantity of larvae ranged from 0.02 to 724.27 per mg of tissue (mean of $82.6 \pm 9.2$ s.E.). Infection values by site and sampling period are included in Supplementary Tables S2-S3. Cycle threshold values of all snail samples fell between the highest and lowest $\mathrm{Ct}$ values of the plasmid standards (13.7 and 34.3 respectively). Ct values of snail samples ranged from 16.3 to 30.7 (mean of $20.1 \pm 0.1$ S.E.). Across qPCR runs, standard curve equations were very consistent with an average slope of $-3.421, y$-intercept of $23.129, R^{2}$ of 0.999 and a reaction efficiency of $95.976 \%$. 


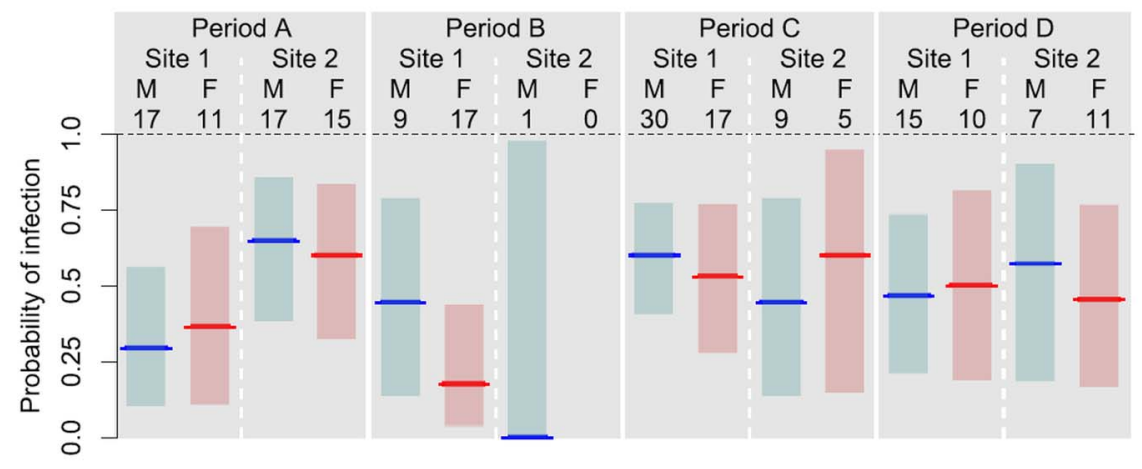

B Prevalence of infection in Rattus exulans

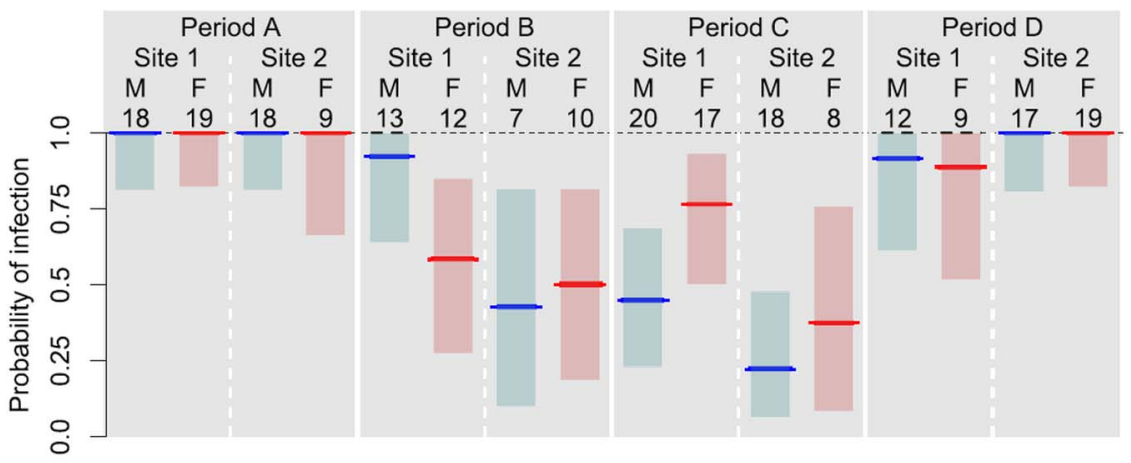

g. 2. Variation in Angiostrongylus cantonensis infection prevalence among sexes, sites and sampling periods for Rattus rattus (A) and $R$. exulans (B) sampled in Hilo, Hawaii. The solid line at the centre of each column depicts the proportion of rats infected in each subsample, irrespective of mass, and the shaded boxes represent the $95 \%$ binomial confidence intervals for the estimate. The number above each sample is the sample size $(N)$. Periods A-D represent host sampling periods (A: May 2018; B: August-September 2018; C: November-December 2018; D: February 2019; with exact dates included in Supplementary Table S1.).

mass was a far more important predictor of infection intensity than length (RVI 0.83 vs 0.11 ) with little influence of their quadratic terms (0.20 and 0.04) (Table 2). The most influential effects were environmental, including site, sampling period and the site $x$ period interaction effect $(0.99,1.00,0.97)$, indicating high variability among sites and sampling periods. We plotted predicted larval counts from the top model (Fig. 6). than the top model). Mass was a far better predictor of infection status than length (RVI $1.0 v s<0.01$ ), with relatively little support for the quadratic term on either $(0.27 v s<0.01)$ (Table 2). The site and sampling period were highly influential on infection status, though only when an interaction term was included, indicating high variability among sites and sampling periods.

Given the high rate of infection observed in P. martensi, with some site/sampling period categories exhibiting $100 \%$ infection ( prevalence $=1.00$ ), regression models could not reliably estimate standard errors for the categorical covariates. Therefore, rather than plot effect sizes from model predictions, we plotted infection prevalence values and $95 \%$ binomial confidence intervals for prevalence by site and sampling period and overlaid the mean \pm the $95 \%$ confidence interval for snail mass (Fig. 4). This plot reflects that a site $\times$ period effect is primarily driven by atypically low prevalence at Site 2 during the February 2019 sampling effort (Period D) and that this low prevalence corresponds to the lowest mean size of snails sampled. Given the problems with predicting confidence intervals from logistic regression including the extrinsic effects, we used regression only to evaluate the influence of snail mass on infection prevalence. Because model selection showed relatively little influence of a quadratic effect of mass $\left(\right.$ mass $^{2}$ ), we simply plot the effect of snail mass on the probability of infection (Fig. 5). From this figure we see a roughly $25 \%$ infection rate in the smallest snails sampled and nearly $100 \%$ by 1.00 $1.50 \mathrm{~g}$ or greater in mass.

\section{Infection intensity in P. martensi}

For P. martensi, the top model for infection intensity was the same as for prevalence (Table $3 \mathrm{C}$ ). As with prevalence, the

\section{Discussion}

In this study, we report on A. cantonensis infection in wild rats ( $R$. exulans and $R$. rattus) and the terrestrial snail, $P$. martensi, in eastern Hawaii Island. An overall infection prevalence of $86.2 \%$ was observed in $P$. martensi and $63.8 \%$ in rats, with R. exulans $(77.4 \%)$ greater than $R$. rattus (47.6\%). Previous reports from eastern Hawaii Island have also shown high infection levels in both rats and snails (Qvarnstrom et al., 2013; Jarvi et al., 2017), (Cowie, 2013; Jarvi et al., 2018; Johnston et al., 2019). Additionally, eastern Hawaii Island is reported to have the greatest area of highly suitable habitat for A. cantonensis in Hawaii, based on current climate conditions (Kim et al., 2019).

We observed high variability in A. cantonensis infection prevalence and intensity for $P$. martensi and $R$. exulans, among the four sampling periods within this study. Interestingly, this effect was not seen for $R$. rattus. Infection levels in $R$. exulans were very high in May 2018 (Period A) and February 2019 (Period D), but generally lower and more variable from the sampling periods in between. For $P$. martensi, we observed a general decreasing trend in infection intensity throughout our sampling period, but only at Site 2, with a less apparent pattern at Site 1 and with moderate variability overall. Variability in infection between sampling period and with no apparent trend. Interestingly, as a result of Hurricane Lane, which occurred from 15 to 29 August 2018 (with heavy rains between dates 22 and 26), over 1000 as well as a high incidence of human infection transmission sites was also seen for $R$. exulans, again strongly influenced by 
Table 3. Model selection tables for the intensity of Angiostrongylus cantonensis infection in rats (Rattus rattus and $R$. exulans) and snails (Parmarion martensi) from two sites over four sampling periods in Hilo, Hawaii.

\begin{tabular}{|c|c|c|c|c|c|}
\hline Model & $\mathrm{df}$ & logLik & $\mathrm{AlC}_{C}$ & $\Delta \mathrm{AIC}_{C}$ & weight \\
\hline \multicolumn{6}{|l|}{ (A) Rattus rattus } \\
\hline mass & 3 & -256.16 & 518.60 & 0.00 & 0.184 \\
\hline mass + site & 4 & -255.11 & 518.69 & 0.09 & 0.175 \\
\hline mass + mass $^{2}$ & 4 & -255.65 & 519.76 & 1.16 & 0.103 \\
\hline mass + mass $^{2}+$ site & 5 & -254.65 & 520.00 & 1.40 & 0.091 \\
\hline period & 5 & -258.52 & 527.74 & 9.14 & 0.002 \\
\hline (null) & 2 & -262.24 & 528.62 & 10.02 & 0.001 \\
\hline site & 3 & -261.99 & 530.26 & 11.66 & 0.001 \\
\hline sex & 3 & -262.23 & 530.73 & 12.13 & 0.000 \\
\hline \multicolumn{6}{|l|}{ (B) Rattus exulans } \\
\hline mass + mass $^{2}+$ site + period + site $\times$ period & 11 & -571.48 & 1166.58 & 0.00 & 0.158 \\
\hline mass + mass $^{2}+$ period & 7 & -575.96 & 1166.59 & 0.01 & 0.157 \\
\hline mass + site + period + site $\times$ period & 10 & -573.13 & 1167.61 & 1.03 & 0.094 \\
\hline mass + mass $^{2}+$ site + period & 8 & -575.79 & 1168.45 & 1.87 & 0.062 \\
\hline mass + period & 6 & -578.02 & 1168.54 & 1.96 & 0.059 \\
\hline mass + mass $^{2}$ & 4 & -580.44 & 1169.12 & 2.54 & 0.044 \\
\hline period & 5 & -583.52 & 1177.39 & 10.81 & 0.001 \\
\hline (null) & 2 & -591.61 & 1187.29 & 20.71 & 0.000 \\
\hline sex & 3 & -590.91 & 1187.96 & 21.38 & 0.000 \\
\hline site & 3 & -591.41 & 1188.95 & 22.37 & 0.000 \\
\hline \multicolumn{6}{|l|}{ (C) Parmarion martensi } \\
\hline mass + site + period + site $\times$ period & 10 & -259.13 & 540.01 & 0.00 & 0.618 \\
\hline period & 7 & -268.98 & 552.82 & 12.82 & 0.001 \\
\hline length & 3 & -274.54 & 555.26 & 15.26 & 0.000 \\
\hline (null) & 2 & -278.06 & 560.20 & 20.20 & 0.000 \\
\hline mass & 3 & -277.68 & 561.53 & 21.53 & 0.000 \\
\hline site & 3 & -277.80 & 561.79 & 21.78 & 0.000 \\
\hline
\end{tabular}

The best models $\left(\triangle \mathrm{AIC} C_{C} \leqslant 2\right)$ are highlighted in bold.

Tables include models that are within $2 \mathrm{AIC}_{C}$ units of the top model (plausible alternative top models, in bold), models including each of the predictor terms alone, and the intercept-only (null) model with no predictor variables; additional combinations of terms were considered, but were unimportant and are not included here for the sake of brevity. Weight = the weight of the respective model in proportion to the sum of all model weights (1.0; Burnham and Anderson, 2002).

mm of rainfall occurred between sampling events for Period B (NNDC CDO, 2020); it is unclear how this influenced our sampling or observations on infection levels.

Although it is difficult to determine information in regards to an annual cycle of infection, since our data only comes from a single year, we do see the prevalence of infection in $R$. exulans return to very high levels in February 2019, similar to that observed in the previous May. These high levels are also consistent with those observed, from the same sites, 2 years prior in February 2017 (Jarvi et al., 2017). One explanation for the temporal differences in infection seen in $R$. exulans could be related to changes in rat-snail interactions due to fluctuations in snail population numbers. If populations of snail species important to A. cantonensis transmission are lower at certain times of the year, a reduction in infections in their rat predators may be occurring as a result, albeit slightly delayed due to the lifecycle of the parasite. Snails are known to go dormant as a response to extremes of heat and drought (Boss, 1974), and in Japan, P. martensi is reported to be more active in the winter (Asato et al., 2004). Anecdotally, we found it much easier to find P. martensi during Periods $\mathrm{C}$ and $\mathrm{D}$ (autumn and winter months).
Additionally, findings from a 2005 survey in eastern Hawaii Island, revealed local residents reporting a population crash of $P$. martensi occurring in the spring with numbers returning to higher levels in the following autumn months (Hollingsworth et al., 2007). More information on temporal changes of abundance and density of host populations, including other intermediate host species, is necessary to accurately describe current predator-prey dynamics and their influence on $A$. cantonensis transmission in the wild. Furthermore, if an annual cycle of infection is occurring in eastern Hawaii, this could have important management or surveillance implications for particular host species in the region.

Here we also report intrinsic factors as strong correlates of $A$. cantonensis infection in all three species investigated. For P. martensi, a positive correlation was observed between body mass and both infection prevalence and intensity, which is likely a result of larger and longer-lived individuals encountering and consuming more food infected with first-stage larvae. While a positive association with $A$. cantonensis infection and $P$. martensi body size, specifically body length, has been previously been shown (Hollingsworth et al., 2007), we found that body mass was a far 


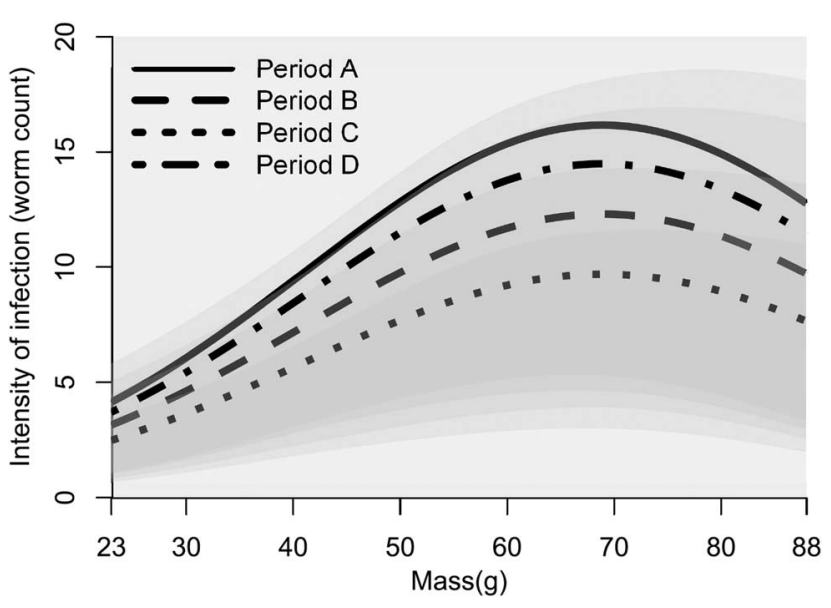

Fig. 3. Model-predicted variation in Angiostrongylus cantonensis infection intensity for Rattus exulans among sampling periods. Shaded areas represent \pm 1 standard error for the estimate; where error intervals broadly overlap, there is little effect size. Periods A-D represent host sampling periods (A: May 2018; B: AugustSeptember 2018; C: November-December 2018; D: February 2019; with exact dates included in Supplementary Table S1.).

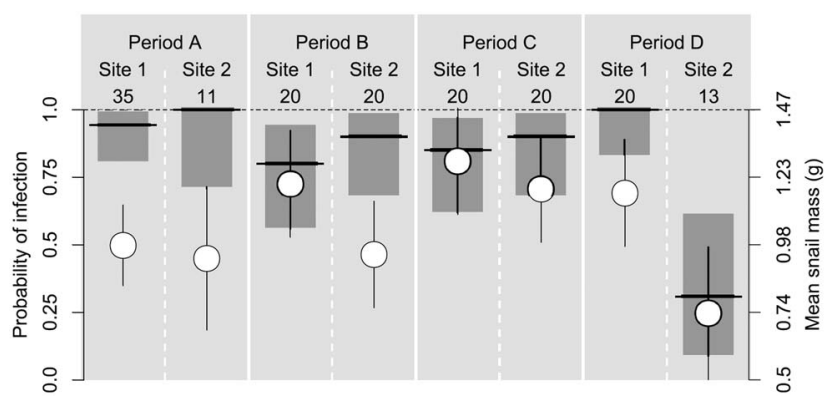

Fig. 4. Prevalence of Angiostrongylus cantonensis infection in Parmarion martensi snails sampled at two sites over four sampling periods in the vicinity of Hilo, Hawaii. Horizontal lines are the prevalence estimates by grouping, with grey bars indicating the $95 \%$ binomial confidence intervals, with values listed on the left $y$-axis. White circles represent the mean mass of snails in the sample, with vertical lines representing the $95 \%$ confidence intervals for the means and values listed on the right $y$-axis. Values above each boxplot indicated the sample size $(N)$. Periods A-D represent host sampling periods (A: May 2018; B: August-September. 2018; C: November-December 2018; D: February 2019; with exact dates included in Supplementary Table S1.).

greater explanatory predictor of infection prevalence and intensity than length. This finding could be related to potential variability in measuring the length of soft-bodied animals and larvae inhabiting the volume of a snail (primarily muscle tissue in $P$. martensi; Asato et al., 2004), which is likely to be more tightly associated with mass, as opposed to any single linear measurement of the body.

Results from our infection analyses in rats were more complex, with different infection trends observed between and within species associated with host body mass and sex. One explanation for these trends could be related to host diet, with higher consumption of infected prey resulting in greater infection (as hypothesized with the intermediate snail hosts). Overall, we observed greater infections in $R$. exulans than in $R$. rattus. Sugihara (1997) found $R$. exulans to have a more restricted diet than $R$. rattus, with a greater relative abundance of invertebrates in the stomachs of $R$. exulans. We also observed intraspecific differences in infections in rats, associated with both host sex and body mass. Infections in $R$. exulans showed a positive correlation with body mass. In $R$. rattus, a negative correlation between infection and body mass was observed, except for infection prevalence in female

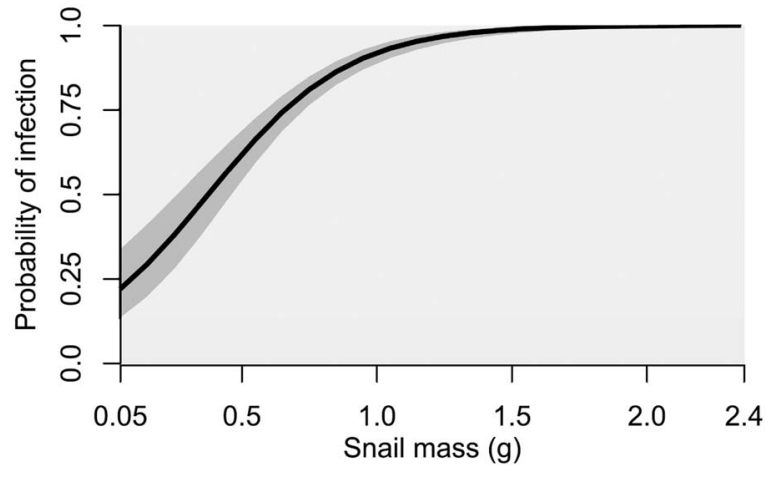

Fig. 5. Model-predicted prevalence of Angiostrongylus cantonensis infection in Parmarion martensi snails sampled over four sampling periods at two sites in Hilo, Hawaii. The shaded area represents \pm 1 standard error of the estimate.

rats. Previous studies on Rattus spp. have also shown that proteinbased food, including invertebrates, was more prominent in the diets of juveniles of both sexes and adult females, as compared to adult males, indicating different diet requirements for growth (age-related) and reproduction (sex-related) (Clark, 1980; Gales, 1982; Sugihara, 1997). While body mass of rodents can fluctuate, in general, body mass in wild rats is positively correlated with age, and is often used as a proxy for age when comparing groups of individuals (Hirata and Nass, 1974; Iskjaer et al., 1989; Webster and Macdonald, 1995; Simões et al., 2014). These differences in feeding patterns could help explain the opposite correlations observed between male and female $R$. rattus for infection prevalence and body mass (i.e. age). However, feeding patterns would not explain the opposite correlations between body mass (i.e. age) and measure of infection (i.e. prevalence and intensity) in female $R$. rattus.

Why might the probability of infection increase with age, while the average worm burden decreases? Furthermore, why would these host sex-specific infection trends not be observed in both rat species sampled here, since they are essentially sharing the same habitat and both were found to be infected? One possibility is that these infection patterns may be related to varying degrees of host immunity to the worms. For rats that are feeding on gastropods in the wild, older individuals are more likely to experience repeated infections over their lifetime than younger individuals. The development of acquired immunity to A. cantonensis has been documented in naturally and experimentally infected Rattus spp., showing lower worm burdens after just a single infection and again in each subsequent infection (Liat et al., 1965; Au and Ko, 1979; Yong and Dobson, 1982). Jarvi et al. (2017) recently hypothesized that in Hawaii, R. rattus, more than R. exulans, are likely developing acquired immunity following primary infections with $A$. cantonensis. They observed a higher prevalence of adult worm infections in $R$. exulans compared to $R$. rattus, but the opposite for third-stage larvae (the stage acquired from the environment), which could be explained by differences in host susceptibility. They also observed opposite correlations between $R$. rattus body mass and prevalence of live adult worms (current infections) and encysted adult worms (previous infections) with the larger (older) rats showing more evidence of past infections and less evidence of current infections than smaller (younger) rats. However, they did not observe the same for $R$. exulans, reporting very high infections levels in older individuals.

Indeed, surveys from the multiple Hawaiian Islands, including this study, have often shown infection levels in $R$. rattus to be lower than $R$. exulans and $R$. norvegicus (Higa et al., 1986; Niebuhr et al., 2019). Interestingly, in our study, we show slight negative trends, between infection and rat body mass, at the 

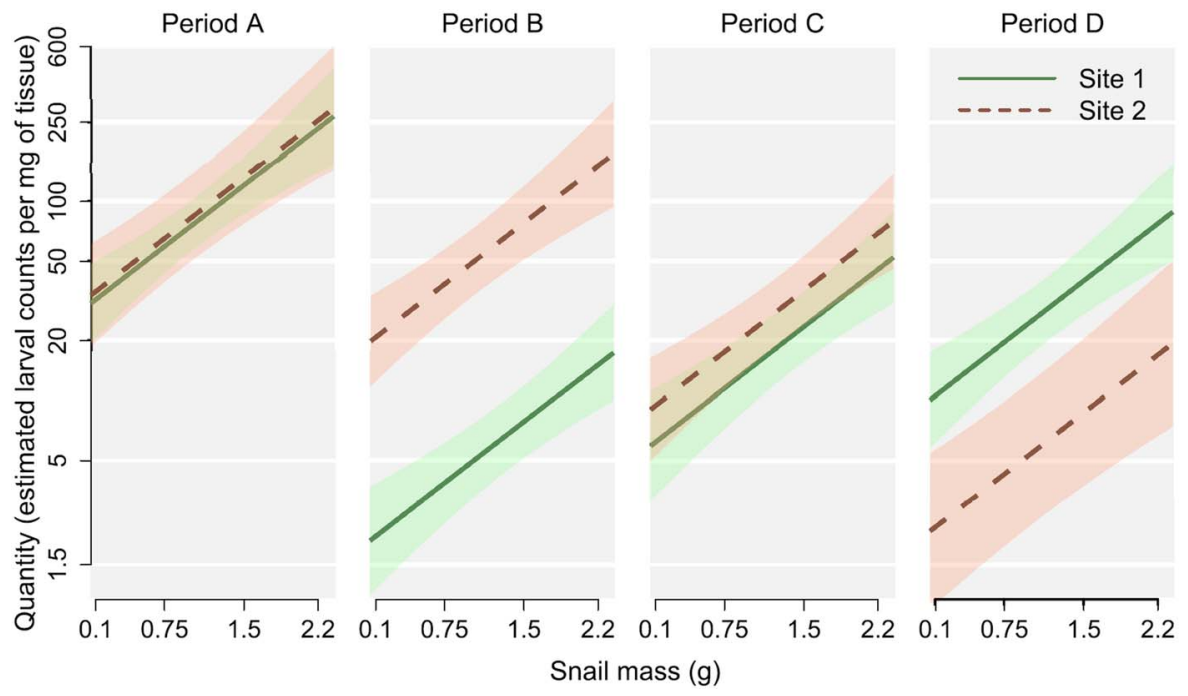

Fig. 6. Intensity of Angiostrongylus martensi infection in Parmarion martensi snails sampled over four sampling periods at two sites in the vicinity of Hilo, Hawaii, as predicted by the top model (mass + site + period + site $x$ period). Shaded areas represent \pm 1 standard error for the estimate; where error intervals broadly overlap, there is little effect size. Periods A-D represent host sampling periods (A: May 2018; B: August-September 2018; C: November-December 2018; D: February 2019; with exact dates included in Supplementary Table S1.).

extreme upper end of body masses for R. exulans, although very few individuals in this body mass range were sampled. It is possible that $R$. exulans is capable of developing some immunity to $A$. cantonensis, but less so than $R$. rattus. It is also possible that a sampling bias occurred for the largest (and oldest) individuals, with more infected rats less successfully trapped due to a decrease in fitness or overall survival. Experimental studies in laboratory strains of Rattus norvegicus have shown that rat survival worsens and body mass decreases with an increase in parasite burden (Ji et al., 2017). Experimental infections are needed to explore the immunity potential of wild rats in Hawaii, which could ultimately inform control operations that may intentionally or unintentionally target certain species, ages or body sizes of rats.

In summary, our results showed that $A$. cantonensis infections in definitive and intermediate hosts in Hawaii are very common overall, but do vary with both environmental and host-related factors. Infection in all species varied, to different degrees, by sampling period and site in ways that did not lend themselves to prediction with a single year of sampling. Host body mass was a strong predictor of infection in the three host species studied here, though the patterns varied between species and sexes in rats. This work also clearly confirms $R$. exulans and P. martensi as important reservoirs of $A$. cantonensis infection in Hawaii. Although these findings cannot clearly illuminate the precise dynamics of host-parasite transmission, they do give a sense of the variability in (1) spatial and temporal expression of A. cantonensis infection prevalence and intensity; and (2) patterns of expression throughout the development of individuals, within definitive and intermediate host populations. Accounting for both predictable and unpredictable sources of variability will be a crucial component in predicting the effectiveness of future disease surveillance or targeted management strategies. Recommendations for future work include multi-year investigations into seasonal and annual infection cycles, to determine any consistency or variation that may be occurring. Additionally, more in-depth spatial infection level data in definitive and intermediate hosts are also needed, including from different locations within and across islands, as well as from different habitat types (e.g. urban, agricultural). More data on variation in host abundance would also be helpful in both informing on transmission cycles among wild populations and designing control operations.

Supplementary material. The supplementary material for this article can be found at https://doi.org/10.1017/S003118202000164X
Acknowledgements. We thank the entire USDA WS NWRC Hawaii Field Station and the UH-Hilo Jarvi Lab for their help with data collection, especially R. Sugihara, T. McAuliffe, N. Preston, D. Sedgewick, A. Deane, K. Snook, J. Jacobs, A. Steel and B. Torres Fischer. Additional thanks to Craig Blaisdell of the State of Hawaii, Department of Defense Environmental Office and to Pana'ewa Rainforest Zoo \& Gardens. Reference herein to any specific commercial products, process, or service by trade name, trademark, manufacturer, or otherwise, does not necessarily constitute or imply its endorsement, recommendation, or favouring by the US Government.

Financial support. This work was supported in part by the US Department of Agriculture, Animal and Plant Health Inspection Service, Wildlife Services, National Wildlife Research Center; the Daniel K. Inouye College of Pharmacy, University of Hawaii at Hilo; the Hawaii State Legislature; a grant from the Hawaii Department of Land and Natural Resources, Hawaii Invasive Species Council; and by Strategic Science Investment Funding from the New Zealand Ministry of Business, Innovation and Employment.

Conflict of interest. None.

Ethical standards. All animal use was reviewed and approved by the USDA WS NWRC Institutional Animal Care and Use Committee under protocol QA-2835.

\section{References}

Alicata JE (1991) The discovery of Angiostrongylus cantonensis as a cause of human eosinophilic meningitis. Parasitology Today 7, 151-153.

Anderson DR (2008) Model Based Inference in the Life Sciences: a Primer on Evidence. New York, New York, USA: Springer Science + Business Media, LLC.

Asato R, Taira K, Nakamura M, Kudaka J, Itokazu $\mathrm{K}$ and Kawanaka M (2004) Changing epidemiology of Angiostrongyliasis cantonensis in Okinawa Prefecture, Japan. Japanese Journal of Infectious Diseases 57, 184-186.

Au ACS and Ko RC (1979) Changes in worm burden, haematological and serological response in rats after single and multiple Angiostrongylus cantonensis infections. Parasitology Research 58, 233-242.

Barratt J, Chan D, Sandaradura I, Malik R, Spielman D, Lee R, Marriott D, Harkness J, Ellis J and Stark D (2016) Angiostrongylus cantonensis: a review of its distribution, molecular biology and clinical significance as a human pathogen. Parasitology 143, 1087-1118.

Boss KJ (1974) Oblomovism in the Mollusca. Transactions of the American Microscopical Society 93, 460-481.

Burnham KP and Anderson DR (2002) Model Selection and Multimodel Inference: a Practical Information-Theoretic Approach, 2nd Edn., New York, New York, USA: Springer-Verlag.

Bustin SA, Benes V, Garson JA, Hellemans J, Huggett J, Kubista M, Mueller R, Nolan T, Pfaffl MW, Shipley GL, Vandesompele J and Wittwer CT (2009) The MIQE guidelines: minimum information for 
publication of quantitative real-time PCR experiments. Clinical Chemistry 55, 611-622.

Červená B, Modrý D, Fecková B, Hrazdilová K, Foronda P, Alonso AM, Lee R, Walker J, Niebuhr CN, Malik R and Šlapeta J (2019) Low diversity of Angiostrongylus cantonensis complete mitochondrial DNA sequences from Australia, Hawaii, French Polynesia and the Canary Islands revealed using whole genome next-generation sequencing. Parasites \& Vectors 12, 241.

Clark D (1980) Age-and sex-dependent foraging strategies of a small mammalian omnivore. The Journal of Animal Ecology 49, 549-563.

Cowie RH (2013) Pathways for transmission of angiostrongyliasis and the risk of disease associated with them. Hawaii Journal of Medicine \& Public Health 72, 70-74

Gales RP (1982) Age- and sex-related differences in diet selection by Rattus Rattus on Stewart Island, New Zealand. New Zealand Journal of Zoology 9, 463-466

Graeff-Teixeira C, Da Silva ACA and Yoshimura K (2009) Update on eosinophilic meningoencephalitis and its clinical relevance. Clinical Microbiology Reviews 22, 322-348.

Higa HH, Brock JA and Palumbo NE (1986) Occurrence of Angiostrongylus cantonensis in rodents, intermediate and paratenic hosts on the island of Oahu. Journal of Environmental Health 48, 319-323.

Hirata DN and Nass RD (1974) Growth and sexual maturation of laboratory-reared, wild Rattus norvegicus, $R$. rattus, and $R$. exulans in Hawaii. Journal of Mammalogy 55, 472-474.

Hollingsworth RG, Kaneta R, Sullivan JJ, Bishop HS, Qvarnstrom Y, DaSilva AJ and Robinson DG (2007) Distribution of Parmarion Cf. martensi (Pulmonata: Helicarionidae), a new semi-slug pest on Hawai'i Island, and its potential as a vector for human angiostrongyliasis. Pacific Science 61, 457-467.

Howe K and Jarvi SI (2017) Angiostrongyliasis (rat lungworm disease): viewpoints from Hawaii Island. ACS Chemical Neuroscience 8, 1820-1822.

Howe K, Kaluna L, Fisher BT, Tagami Y and Mchugh R (2019) Water transmission potential of Angiostrongylus cantonensis: larval viability and effectiveness of rainwater catchment sediment filters. PLOS ONE 14, e0209813.

Ibrahim MM (2007) Prevalence and intensity of Angiostrongylus cantonensis in freshwater snails in relation to some ecological and biological factors. Parasite 14, 61-70.

Innes JG (1990) Ship rat. In King CM (ed.), The Handbook of New Zealand Mammals. Auckland, NZ: Oxford University Press, pp. 206-225.

Iskjaer C, Slade NA, Childs JE, Glass GE and Korch GW (1989) Body mass as a measure of body size in small mammals. Journal of Mammalogy 70, 662-667.

Jarvi SI, Farias MEM, Howe K, Jacquier S, Hollingsworth R and Pitt W (2012) Quantitative PCR estimates Angiostrongylus cantonensis (rat lungworm) infection levels in semi-slugs (Parmarion Martensi). Molecular and Biochemical Parasitology 185, 174-176.

Jarvi SI, Quarta S, Jacquier S, Howe K, Bicakci D, Dasalla C, Lovesy N, Snook K, McHugh R and Niebuhr CN (2017) High prevalence of Angiostrongylus cantonensis (rat lungworm) on eastern Hawai'i Island: a closer look at life cycle traits and patterns of infection in wild rats (Rattus Spp.). PLoS ONE 12, e0189458.

Jarvi SI, Howe K and Macomber P (2018) Angiostrongyliasis or rat lungworm disease: a perspective from Hawai i. Current Tropical Medicine Reports 5, 59-66.
Ji L, Yiyue X, Xujin H, Minghui Z, Mengying Z, Yue H, Yanqi W, Langui S, Xin Z, Datao L, Shuo W, Huanqin Z, Zhongdao W and Zhiyue L (2017) Study on the tolerance and adaptation of rats to Angiostrongylus cantonensis infection. Parasitology Research 116, 1937-1945.

Johnston DI, Dixon MC, Elm JL, Calimlim PS, Sciulli RH and Park SY (2019) Review of Cases of Angiostrongyliasis in Hawaii, 2007-2017. The American Journal of Tropical Medicine and Hygiene 101, 608-616.

Kim JR, Hayes KA, Yeung NW and Cowie RH (2014) Diverse gastropod hosts of Angiostrongylus cantonensis, the rat lungworm, globally and with a focus on the Hawaiian Islands. PLoS ONE 9, 21-26.

Kim JR, Wong TM, Curry PA, Yeung NW, Hayes KA and Cowie RH (2019) Modelling the distribution in Hawaii of Angiostrongylus cantonensis (rat lungworm) in its gastropod hosts. Parasitology 146, 42-49.

Liat LB, Kong OC and Joe LK (1965) Natural infection of Angiostrongylus cantonensis in Malaysian rodents and intermediate hosts and preliminary observations on acquired resistance. American Journal of Tropical Medicine and Hygiene 14, 610-621.

Niebuhr CN, Jarvi SI and Siers SR (2019) A review of rat lungworm infection and recent data on its definitive hosts in Hawaii. Human-Wildlife Interactions 13, 238-249.

Niebuhr CN, Jarvi SI, Kaluna L, Fischer BLT, Deane AR, Leinbach IL and Siers SR (2020) Occurrence of rat lungworm (Angiostrongylus cantonensis) in invasive coqui frogs (Eleutherodactylus Coqui) and other hosts in Hawaii. Journal of Wildlife Diseases 56, 203-207.

NNDC CDO (2020) NOAA National Data Center Climate Data Online. National Climatic Data Center, Asheville, NC, USA. Available at http:// cdo.ncdc.noaa.gov/CDO/cdo (Accessed 15 April 2020).

Prociv P, Spratt DM and Carlisle MS (2000) Neuro-angiostrongyliasis: unresolved issues. International Journal for Parasitology 30, 1295-1303.

Qvarnstrom Y, Bishop HS and da Silva AJ (2013) Detection of rat lungworm in intermediate, definitive, and paratenic hosts obtained from environmental sources. Hawaii Journal of Medicine \& Public Health 72(suppl. 2), 63-69.

R Core Team (2019) R: A Language and Environment for Statistical Computing. $R$ Foundation for Statistical Computing. Vienna, Austria: $\mathrm{R}$ Foundation for Statistical Computing.

Simões RO, Maldonado Júnior A, Olifiers N, Garcia JS, Bertolino AVFA and Luque JL (2014) A longitudinal study of Angiostrongylus cantonensis in an urban population of Rattus norvegicus in Brazil: the influences of seasonality and host features on the pattern of infection. Parasites of Vectors $7,1-8$.

Spratt DM (2015) Species of Angiostrongylus (Nematoda: Metastrongyloidea) in wildlife: a review. International Journal for Parasitology: Parasites and Wildlife 4, 178-189.

Sugihara RT (1997) Abundance and diets of rats in two native Hawaiian forests. Pacific Science 51, 189-198.

Wang QP, Lai DH, Zhu XQ, Chen XG and Lun ZR (2008) Human angiostrongyliasis. The Lancet Infectious Diseases 8, 621-630.

Webster JP and Macdonald DW (1995) Parasites of wild brown rats (Rattus norvegicus) on UK farms. Parasitology 111, 247-255.

Yong WK and Dobson CM (1982) The biology of Angiostrongylus cantonensis larvae in immune rats. Southeast Asian Journal of Tropical Medicine and Public Health 13, 244-248.

York EM, Creecy JP, Lord WD and Caire W (2015) Geographic range expansion for the rat lungworm in North America. Emerging Infectious Diseases 21, 1234-1236. 